\title{
Ecological Teardown
}

\author{
RHETT RUSSO \\ Rensselaer Polytechnic Institute
}

In Being Ecological, Timothy Morton proposes that being ecological is not a simply matter of exercising our free will as humans. The premise that there is a firewall which separates us from the rest of the world is an ontological impossibility. Foremost, being ecological is a process of learning to be attuned to the world. Similarly, Latour's 'compositionist' narrative identifies a world that presents 'ever increasing intimacy with new natures.' Intimacy requires acceptance and care and both are matters of designing how we choose to live with technology. The studio work presented here focuses on the design of homes that seek to attune energy production to domestic life through aesthetics. The studio is initiated through an examination of the photographic teardowns of Todd McLellan. The photographs include a variety of handheld domestic products that have been completely disassembled into two-dimensional archives of anthropomorphic, mechanical and digital components. Torn down, and emptied out, each object provides students with a chronicle for designing the future.

To succeed, an ecological politics must manage to be at least as powerful as the modernizing story of emancipation without imagining that we are emancipating ourselves from Nature. What the emancipation narrative points to as proof of increasing human mastery over and freedom from Natureagriculture, fossil energy, technology-can be redescribed as the increasing attachments between things and people at an ever-expanding scale. If the older narratives imagined humans either fell from Nature or freed themselves from it, the compositionist narrative describes our ever-increasing degree of intimacy with the new natures we are constantly creating. Only "out of Nature" may ecological politics start again and anew.

-Bruno Latour, Love Your Monsters. Why We Must Care for Our Technologies As We Do Our Children

\section{PHOTOGRAPHIC REALISM AND ATTUNEMENT}

Contemporary photography is unique in its ability to capture, in the fraction of a second, the fleeting images of the real world, along with the capability to amend that world, digitally. Todd McLellan's teardowns, Adam Magyar's slit photography, Filip Dujardin's Fictions, and Andreas Gefeller's, Japan Series, all use photographic realism as a means of presenting what is ordinary to us from a new perspective. Their work is unique in its ability to confront the viewer with an aesthetic experience of the everyday and to achieve it through a high level of technical refinement. These artists use the medium of digital photography to explore images that synthesize veracity - the accurate, and the credible aspect of photography, with fiction (Fontcuberta 122). Over the last several years the work of these photographers has served as a basis for introducing architecture students to ecology and aesthetics through the ontological framework of photographic realism. If the strength of abstraction is its ability to defer signification or blur the identity of things, realism presents the qualities of things head on. As case studies, these photographs have helped illustrate how to combine these two design approaches to alter our perception of the everyday.

\section{TEARING DOWN}

The first objective in the studio is to have the students encounter veracity firsthand. To achieve this the students are tasked with making a digital 'reconstruction' of one Todd McLellan's teardown photographs and to represent the field of components from an alternate viewpoint (figure1). Everything must be collected, from color, finishes, materials, to scratches, and screws, and the students are encouraged to scavenge the web for inventories and replacement parts. McLellan has meticulously disassembled and photographed over fifty domestic objects, encompassing one hundred years of product design evolution. Categorized according to their relative size, the teardowns expose the inner workings of items such as the Gerhard Heintzman Upright Piano (1911) that tops out with 1842 components, to the Rotring mechanical pencil (1997) with a mere 16 components, a Northern Electric Telephone (1980s), CHI Hair Straighteners (1989), a LACIE Hardrive (2000s), a Bissel Carpet Steamer (2005), and the iPad 2 (2011). While the objects are no longer mechanically, spatially and physically operational the photographs expose the alienation of the componentry that has transpired as the gears and screws of mechanical engineering have been superseded by the circuits and glues of digital technology. The teardowns attune the students to more than what is simply inside the black box, they allow each student to develop a solidarity with a nonhuman thing (Morton, 73). By comprehending the double-sidedness of objects that are embedded with anthropomorphic grips, ventilation holes, branding, electrical contacts and 


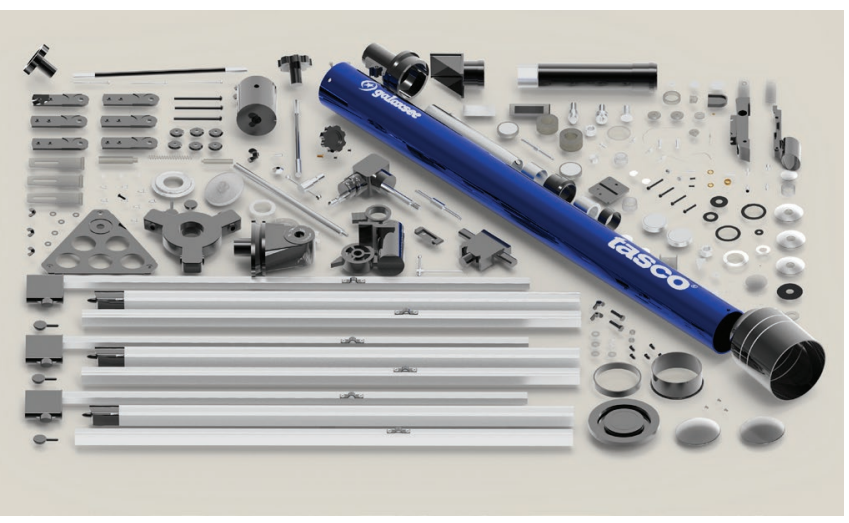

Figure 1. Amatullah Kose, Digital reconstruction of the 'Telescope 2000 Tasco Component count: 223,' in Todd McLellan's, Things Come Apart, AD 2, 2019, Rennselaer Polytechnic Institute (Photo by author).

structural flanges exposes the influences to a hybrid world of design. This understanding sheds light on the compactness of things -and the encryption- that exists in the design of tools and instruments which bridge between the human and non-human world.

\section{GROKING \& ENERGY}

Tasked with designing a house and equipped with a collection of components the second objective is to begin to reaffirm what the objects share with architecture. During this phase of the design the students are asked to reassemble the parts or features of the object to construct a model. This phase of design takes it que from the teardown and the inability to read how the object goes back together. According to Morton our experience with objects never allows us to grasp them in the present, this remains beyond access, rather objects are revealed to us in slivers through their past and in the future. After a myriad of trials and errors, affinities for certain features develop, and the parts begin to be reconnected (figure 2).

Nested within McClellan's monograph are five texts that identify cultural phenomena that concern the ecology of industrial products. The texts are written by authors from different disciplines, including forensic restoration, maker culture, repair culture, and industrial design. One of the more unusual terms that is introduced is groking, which was coined in the 1961 science fiction film, A Stranger in a Strange Land, by Robert Heinlein in which he describes a world of aliens living in Mars. In Heinlein's sense of the word, groking means 'identically equal' (McGiveron). This sensibility is also part of repairing consumer objects. As Kyle Wiens writes, In The Repair Revolution, "Once you grok your possessions, a world of possibilities opens up. Knowing how a thing works enables you to adapt it." Repair culture relies on developing empathy for things we are unfamiliar with by becoming immersed in them. In other words, if we form bonds with non-human things

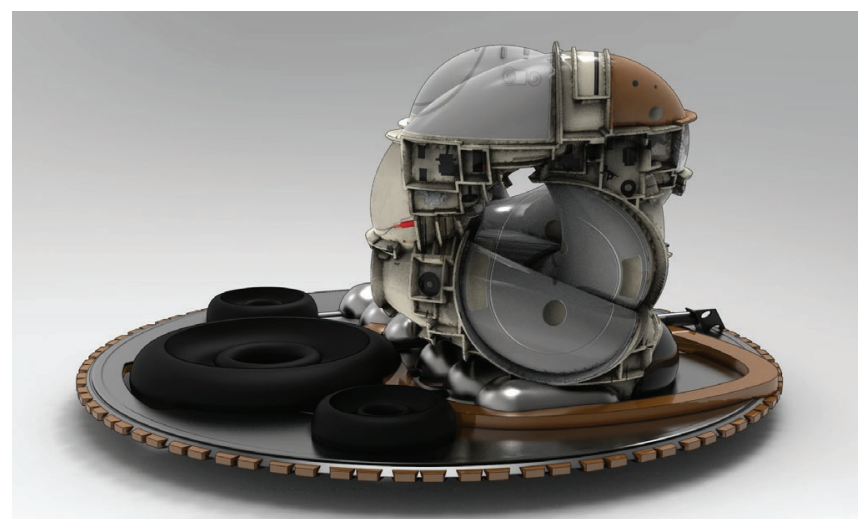

Figure 2. Aida Ayuk. A House of Ballooning Bio-Gas, on a Turntable Garden, AD 2, Rennselaer Polytechnic Institute, 2019 (Photo by author).

(Morton's uses the word care) only then are we are then able to love or hate them. What is interesting about this format is that repairing something demands coming to terms with what you don't know about it and in some circumstances this may result in repairing something in such an absurd manner that it changes our expectations. Groking is a form of attunement that involves taking an alien (a domestic product) from the past and proceeds by making it a stranger in the future. What the studio has discovered is that groking an object alters its ecology in such a way that it reveals a second life.

To explore this further the studio has adopted a method of designing the house using a particular energy format, such as hydropower, buoyant air turbines, photo-voltaic, photobioreactors, biomeilers, bio-gas digesters, dew catchers, and various forms of geothermal energy. These systems serve as a blueprint for reintroducing the flow of energy to the products and their environments at the scale of architecture. In simple terms, they serve as re-animators for an architectural encounter. Through this process of storing and transforming energy, the disciplinary categories of wall, roof, drainage, envelope, floor and comfort have to be carefully considered (figure 3 ). What has developed is a design process that allows students to tinker with the architecture through the adversarial presence of domestic energy and its impact on the adjacent landscape (figure 5). It is through this attunement with technology and domestic occupation that the studio is able to return to Latour's provocation of the compositionist.

Tinkering also plays an important role in this process by providing a format for speculation. To tinker is to rewrite the use value of a thing to serve another purpose. These encounters provide safe spaces where things break or reveal hidden potentials of things that cannot be reduced to their parts. As one author describes the urgency of fashioning of a new wheel for a cart, 'where duct tape 

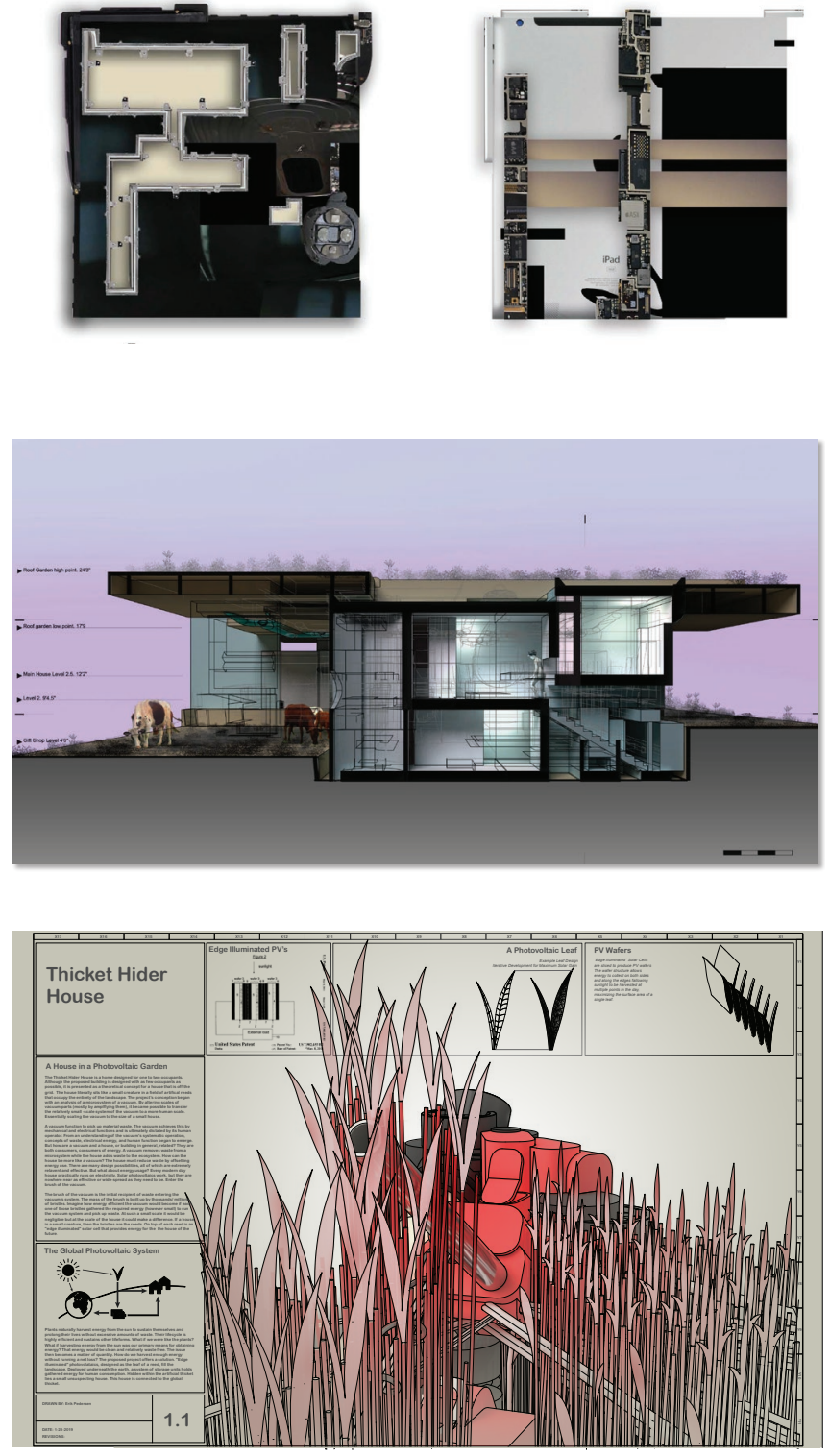

Figure 3. Top and middle, Katie Hoffstadter, domestic roof for processing methane for the Bio-gas House, AD2, Rennselaer Polytechnic Institute, 2017; bottom, Erik Pedersen, Thicket Hider House, domestic energy from a photovoltaic garden. AD 2, Rennselaer Polytechnic Institute, 2018, PV patent by Partha Dutta (Photos by author).

becomes a wheel that lasted about 3 miles.' (Tulley 78). While these general categories represent a framework of caring for technology - tearing down, groking, tinkering, restoring and joining each engender their own aesthetic sensibility for putting architecture together through the presence of objects.

It is in this spirit that the studio begins to identify certain aspects of the product's anatomy with tangible slivers of landscape, house and energy. Its's important to note that Heinlein's concept of groking or being 'identically equal' serves as vehicle for identifying how features of the products help to expose the slivers of reality within the landscapes, houses and the flows of energy that are otherwise inaccessible to us as designers of the built environment. The design approach at this phase is more often visual rather than causal and it precipitates through the vicariousness of the objects themselves by encouraging the students to perceive and encounter things in a new way. As Timothy Morton and Jane Bennett have pointed out granting rights to nonhuman things such as beaver dams, coral reefs, soil biomes, and rainforests first requires us to recognize them as beings.

\section{ENERGY PATENT-SHEETS}

The photographs present a hidden world of wires, chips, circuits, screws, amplifiers that are equally dysfunctional and flat in relation to each other, yet by virtue of McLellan's painstaking labor to confine all of the parts to fit into a rectangle, he says, 'I stayed faithful to the assembly and tried to lay out the pieces in the order in which they were revealed...almost like a family portrait' (McLellan 11). This statement reflects the importance of the systems that are locked into the objects and an acknowledgement that there is an order to time, space and material that can be captured through photography. We are faced with a similar reality when it comes to the design of architecture. To address this the students are asked to develop a patent-sheet for the home based upon their energy system. These sheets are intended to help format how and where energy flows through the house. The sheets combine rules of thumb, data and patents, to help identify where and what scale technology overlaps with domestic life and the space requirements of the house (figure 4).

The patent-sheets provide an opportunity to teach students how to couple and uncouple materiality from form in the design process. Often, the survey of architectural precedents is inadequate to convey the divergent approaches to assigning material in the design and finish of buildings. The vast number of different materials that exist inside of a consumer product is not dissimilar to what we might find in a building. As systems based diagrams or sections, the patent-sheets help unpack the array of producers, consumers, circuits, storage, and amplifiers, that have been carefully nested within each object. The veracity of the proposals rests on how the home becomes a collection of things. What is unique about this perspective is that it reveals that even the smallest things are not designed in isolation. Buttonholes, borings, and bossings are part of the tuning and the empathy that emerges as things comingle. The teardowns have proved invaluable when it comes to introducing materiality, disassembly and tectonics in the design studio and they attune the students to the power, veracity and impact of how we interact with objects to form architecture. 

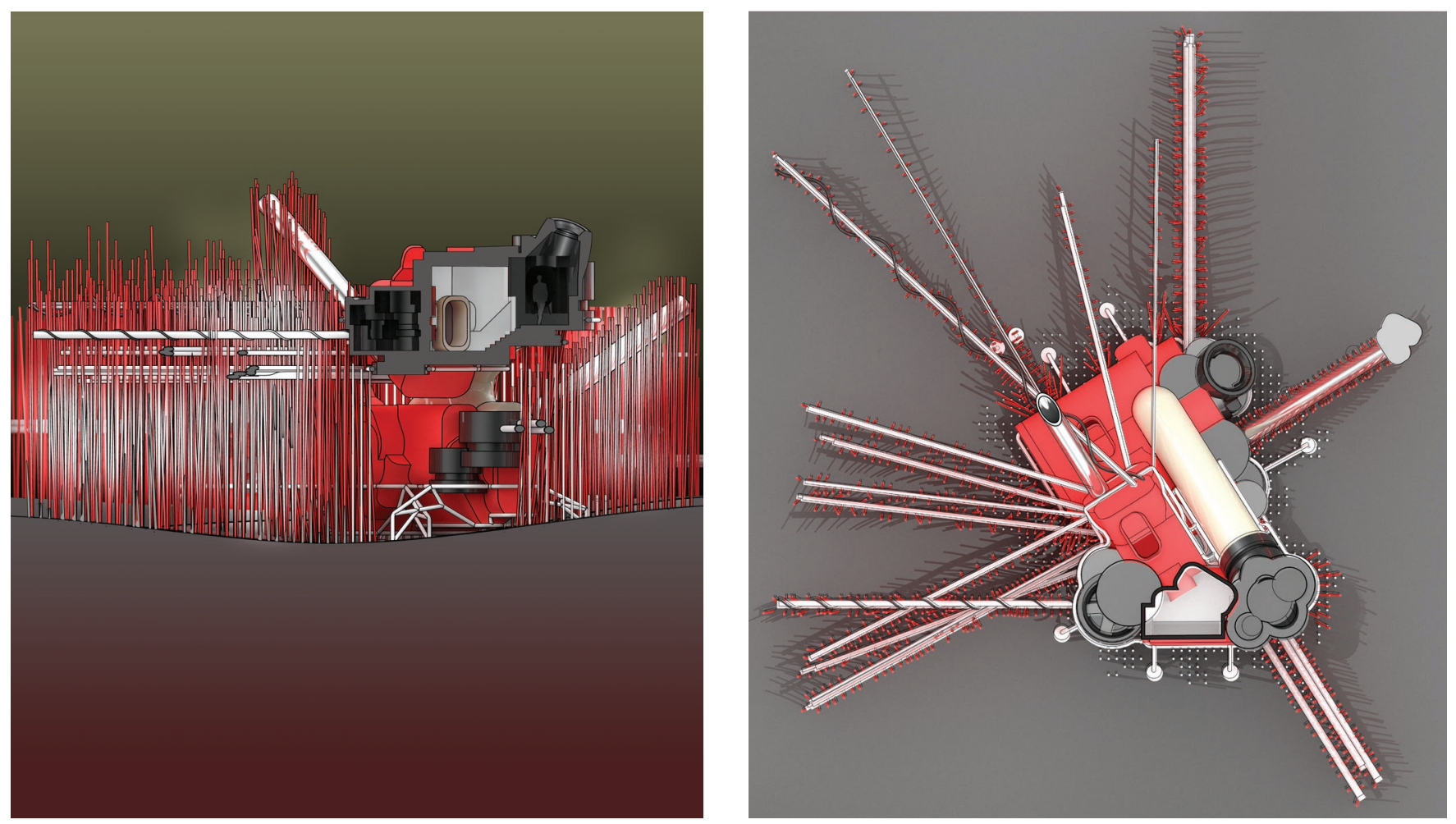

Figure 4. Erik Pedersen, Thicket Hider House, site section and upper level plan, AD2 Rennselaer Polytechnic Institute, 2018 (Photos by author).

Acknowledgements: I would like to gratefully acknowledge the leadership of Professor Anthony Titus for his development of the first year curriculum at RPI. Without his curation and openness, this pathway would not have been possible.

\section{REFERENCES}

Bendall, Penny. "Taking Apart The Past," in Things Come Apart: A Teardown Manual For Modern Living. New York: Thames \& Hudson, 2013.

McGiveron, Rafeeq O. “From Free Love to the Free-Fire Zone: Heinlein's Mars, 19391987," Extrapolation 42, no. 2 (Summer 2001): 137-149.

McLellan, Todd. Things Come Apart: A Teardown Manual For Modern Living. New York: Thames \& Hudson, 2013.

Morton, Timothy. Being Ecological. Cambridge, MA: The MIT Press, 2018.

Tulley, Gever. "Life Lessons Learned Through Tinkering," in Things Come Apart: A Teardown Manual For Modern Living. New York: Thames \& Hudson, 2013.

Wiens, Kyle. "The Repair Revolution" in Things Come Apart: A Teardown Manual For Modern Living. New York: Thames \& Hudson, 2013. 